\title{
STABLE PROCESSES WITH STATIONARY INCREMENTS PARAMETERIZED BY METRIC SPACES
}

\author{
ZUOPENG FU AND YIZAO WANG
}

\begin{abstract}
A new family of stable processes indexed by metric spaces with stationary increments are introduced. They are special cases of a new family of set-indexed stable processes with Chentsov representation. At the heart of the representation, a result on the so-called measure definite kernels is of independent interest. A limit theorem for set-indexed processes is also established.
\end{abstract}

\section{INTRODUCTION}

A stochastic process is most commonly referred to as a time-indexed collection of random variables. However, stochastic processes indexed by other generic sets, sometimes referred to as parameterized by metric spaces, also have a long history in probability theory. The probabilistic properties of stochastic processes are intrinsically connected to the geometry of the metric space, and the interactions can be very rich. See for example [1, 2, 25], just to mention a few.

Our motivating example is the Brownian motion. Paul Lévy first introduced in the late 40s the notion of a Brownian motion indexed by a metric space, denoted by $(\mathrm{M}, \mathrm{d})$ throughout. When the Brownian motion, say $\left\{\mathbb{B}_{x}\right\}_{x \in \mathrm{M}}$, exists, it is a centered Gaussian process determined by $\mathbb{B}_{o}=0$ for a marked point $o \in \mathrm{M}$ and

$$
\mathbb{E}\left(\mathbb{B}_{x}-\mathbb{B}_{y}\right)^{2}=\mathrm{d}(x, y) \text {, for all } x, y \in \mathrm{M} .
$$

Under the assumption $\mathbb{B}_{o}=0$, the above is equivalent to

$$
\operatorname{Cov}\left(\mathbb{B}_{x}, \mathbb{B}_{y}\right)=\frac{1}{2}(\mathrm{~d}(x, o)+\mathrm{d}(y, o)-\mathrm{d}(x, y)) .
$$

Such a process is known as a Lévy Brownian field 24]. Lévy first considered the case of $\mathrm{M}=\mathbb{S}^{n}$ (the Euclidean sphere), then Chentsov [40] addressed the case of $\mathrm{M}=\mathbb{R}^{n}$ and then Molchan 26] investigated the case of $\mathrm{M}=\mathbb{H}^{n}$ (the hyperbolic space) and more general symmetric spaces. Since the so-defined processes are Gaussian, a necessary and sufficient condition for the existence is for the metric $\mathrm{d}$ to be of conditionally negative type [12]. Nevertheless, corresponding integral representations of Lévy Brownian fields (i.e. stochastic integrals with respect to a white noise) have been developed too. In particular, Takenaka et al. [39] explained a general framework in terms of projective geometry that provides Chentsov-type integral representations for Lévy Brownian fields indexed by $\mathbb{R}^{n}, \mathbb{S}^{n}$ and $\mathbb{H}^{n}$. We shall focus on stochastic processes parameterized by these three metric spaces in

Date: May 3, 2019.

2010 Mathematics Subject Classification. 60G22, 60G52; Secondary, 60G60.

Key words and phrases. Lévy Brownian field, set-indexed process, stable process, stationary increment, Chentsov representation, measure definite kernel. 
this paper. In general, if the index manifold is not simply connected, then a Lévy Brownian field does not exist [41].

There is a lately renewed interest of investigating fractional stochastic processes indexed by metric spaces. By fractional stochastic processes, we consider extensions of fractional Brownian motions, as stochastic processes indexed by $\mathbb{R}_{+}=[0, \infty)$, to Gaussian processes indexed by generic metric spaces, and further to stable processes 8, 19, 38]. For extensions of Lévy Brownian fields, as a natural extension of the fractional Brownian motions indexed by $\mathbb{R}_{+}$, we name a centered Gaussian process $\left\{\mathbb{B}_{x}^{H}\right\}_{x \in \mathrm{M}}$ a fractional Lévy Brownian field, as long as

$$
\operatorname{Cov}\left(\mathbb{B}_{x}^{H}, \mathbb{B}_{y}^{H}\right)=\frac{1}{2}\left(\mathrm{~d}^{\beta}(x, o)+\mathrm{d}^{\beta}(y, o)-\mathrm{d}^{\beta}(x, y)\right) \quad \text { with } \quad H=\beta / 2,
$$

and $\mathbb{B}_{o}^{H}=0$ for some $o \in \mathrm{M}$, or equivalently $\mathbb{E}\left(\mathbb{B}_{x}^{H}-\mathbb{B}_{y}^{H}\right)^{2}=\mathrm{d}^{\beta}(x, y)$. Note that the right-hand side of (1.1) a priori is not a valid covariance function for all $\beta>0$. Such a framework has been recently considered by Istas [17], and the legitimate ranges for $\beta$ are known to be intervals in the form of $\left(0, \beta_{\mathbf{M}}\right]$, with $\beta_{\mathbb{R}^{n}}=2, \beta_{\mathbb{S}^{n}}=\beta_{\mathbb{H}^{n}}=1$. It is worth noticing that for $\mathbb{S}^{n}$ and $\mathbb{H}^{n}$, the extension of fractional Lévy Brownian fields only exists for $H \in(0,1 / 2]$. Each fractional Lévy Brownian field (1.1) also has stationary increments with respect to a certain group action on $\mathrm{M}$, see (2.2) below.

Recent developments on fractional Lévy Brownian fields include for example 7, 21] (indexed by $\mathbb{S}^{n}$ ). More generally, in the spatial context a Gaussian process $\left\{G_{x}\right\}_{x \in \mathrm{M}}$ is often characterized by its variogram $v(x, y):=\mathbb{E}\left(G_{x}-G_{y}\right)^{2}$ (then stationary increments imply that $v(x, y)$ is a function of $\mathrm{d}(x, y))$, and there is already a huge literature on random fields from this aspect; see for example [3, 42] for latest surveys on Gaussian random fields. Other types of generalizations of fractional Brownian motions include for example [15, 27].

Much fewer examples have been known for non-Gaussian stable processes indexed by metric spaces. We are in particular motivated by examples of such processes with stationary increments. A natural extension of (1.1) to stable processes, say $\left\{Z_{x}\right\}_{x \in \mathrm{M}}$, would require necessarily that

$$
\frac{Z_{x}-Z_{y}}{\mathrm{~d}^{\beta / \alpha}(x, y)} \sim S_{\alpha}(\sigma, 0,0), \text { for all } x, y \in \mathrm{M},
$$

where the right-hand side stands for symmetric $\alpha$-stable $(\mathrm{S} \alpha \mathrm{S})$ distribution with scale parameter $\sigma>0$. The above relates the increments of the process and the geodesic distance in a unified manner as in the Gaussian case. However for $\alpha \in$ $(0,2)$, unlike the Gaussian case $\alpha=2,(1.2)$ is a strictly weaker notion than selfsimilarity and stationary increments even for $M=\mathbb{R}_{+}$, and is satisfied by different stable processes. Many examples of self-similar stable processes with stationary increments exist for $M=\mathbb{R}_{+}[29$, 35]. In contrast, for other choices of $M$, only the following examples are known to have stationary increments and satisfy (1.2):

a. Lévy-Chentsov stable fields [38] as natural extensions of Lévy Brownian fields sharing the same Chentsov representations (to be reviewed in Section 2.2),

b. Lévy-Chentsov sub-stable fields (to be reviewed in Section 2.3. revisited recently in [18]), and

c. Takenaka stable fields [37] for $\mathrm{M}=\mathbb{R}^{n}$ only (see also [35, Chapter 8.4]).

The main contributions of the paper are as follows. 
1. A new family of stable processes, referred to as fractional Lévy-Chentsov stable fields, are introduced, as an extension of fractional Lévy Brownian fields. These are $\mathrm{S} \alpha \mathrm{S}$ processes indexed by $\mathrm{M}=\mathbb{R}^{n}, \mathbb{S}^{n}, \mathbb{H}^{n}, \alpha \in(0,2]$, and are shown to have stationary increments and also satisfy (1.2) with $\beta \in(0,1)$ (Theorem 3.4). They have Chentsov-type integral representation, which for $\alpha=2$ is new for fractional Lévy Brownian fields. More generally, these processes are special cases of setindexed Karlin stable processes that we shall introduce.

2. At the core of our presentation, a result on the so-called measure definite kernels [34] is of its own interest from analysis point of view. Recall that a metric $d$ is a measure definite kernel, if there exists a measure space $(E, \mathcal{E}, \mu)$ and a family of sets $A_{x} \in \mathcal{E}$ for all $x \in \mathrm{M}$, such that

$$
\mathrm{d}(x, y)=\mu\left(A_{x} \Delta A_{y}\right) \text {, for all } x, y \in \mathrm{M} .
$$

This property is strictly stronger than that $d$ is of conditionally negative type, and has already been used in the Chentsov representation of Lévy Brownian fields (see e.g. 19, 39]). (This property is also a special case of the Crofton formulae in integral geometry [33, 36].) Here, it is shown that if $d$ is a measure definite kernel, then for all $\beta \in(0,1)$, so is $\mathrm{d}^{\beta}$ with respect to a different measure space and sets $\left\{A_{x}^{*}\right\}_{x \in \mathrm{M}}$ (Proposition 4.1). Based on this result, it follows immediately that fractional Lévy-Chentsov stable fields satisfy (1.2) (Proposition 3.5).

3. A limit theorem is established for set-indexed Karlin stable processes (Theorem [5.1), as a generalization of recent developments on the Karlin model [9, 10], an infinite urn scheme originally considered by Karlin [20] (see also [13]).

The paper is organized as follows. Section 2 reviews Lévy-Chentsov stable fields and introduces the notations to be used later. Section 3 introduces the set-indexed Karlin stable processes, and fractional Lévy-Chentsov stable fields parameterized by metric spaces. Section 4 explains the key result on measure definite kernels. Section 5 establishes a limit theorem for the general set-indexed Karlin stable processes.

\section{LÉVy-Chentsov STABLE FIELDS}

In this section, we review the notion of Lévy-Chentsov stable fields parameterized by metric spaces [37, 38]. Most results have been known, and the goal here is to present a self-contained and systematic presentation in the framework of Chentsov random fields, which plays a crucial role in the following sections.

2.1. Chentsov representation. Let $(\mathrm{M}, \mathrm{d})$ be a metric space. Later on we shall focus on $\mathrm{M}=\mathbb{R}^{n}, \mathbb{S}^{n}, \mathbb{H}^{n}$, and $\mathrm{d}$ the corresponding geodesic metric. We take the convention throughout that an $\mathrm{S} \alpha \mathrm{S}$ distribution with scale parameter $\sigma>0$, denoted by $S_{\alpha}(\sigma, 0,0)$, has characteristic function $\exp \left(-\sigma^{\alpha}|\theta|^{\alpha}\right)$ for $\alpha \in(0,2]$, including the

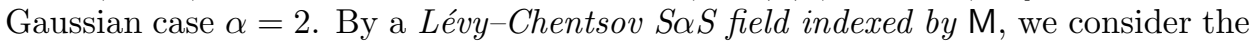
following stable process with Chentsov representation:

$$
Z_{\alpha}(x):=\int_{E} \mathbf{1}_{A_{x}} d M_{\alpha}=M_{\alpha}\left(A_{x}\right), x \in \mathrm{M},
$$

where $M_{\alpha}$ is an $\mathrm{S} \alpha \mathrm{S}$ random measure on a measurable space $(E, \mathcal{E})$ with a $\sigma$-finite control measure $\mu$, for $\alpha \in(0,2]$, and $\left\{A_{x}\right\}_{x \in \mathrm{M}}$ is a collection of elements from $\mathcal{E}$ such that $\mu\left(A_{x}\right)<\infty$. Recall that the random measure $M_{\alpha}$ evaluated at every 
measurable set $A \in \mathcal{E}$, provided $\mu(A)<\infty$, is distributed as $S_{\alpha}\left(\mu(A)^{1 / \alpha}, 0,0\right)$, is $\sigma$ additive over disjoint sets almost surely, and is independently scattered: that is, $M_{\alpha}$ over disjoint sets are independent. Moreover, the characteristic function of finitedimensional distributions of $Z_{\alpha}$ is, for all $d \in \mathbb{N} \equiv\{1,2, \ldots\}, \boldsymbol{\theta} \in \mathbb{R}^{d}, x_{1}, \ldots, x_{d} \in$ $\mathrm{M}, \Lambda^{d}=\{0,1\}^{d} \backslash\{(0, \ldots, 0)\}$,

$$
\mathbb{E} \exp \left(i \sum_{j=1}^{d} \theta_{j} Z_{\alpha}\left(x_{j}\right)\right)=\exp \left(-\sum_{\delta \in \Lambda^{d}}|\langle\boldsymbol{\theta}, \boldsymbol{\delta}\rangle|^{\alpha} \mu\left(\bigcap_{j=1}^{d} A_{x_{j}}^{\delta_{j}}\right)\right),
$$

where $\langle\boldsymbol{\theta}, \boldsymbol{\delta}\rangle=\sum_{j=1}^{d} \theta_{j} \delta_{j}$ and we follow the convention here and below

$$
A^{\delta}=\left\{\begin{array}{ll}
A & \delta=1 \\
A^{c} & \delta=0
\end{array} .\right.
$$

See [35, Chapter 8.2] for more on Chentsov random fields indexed by $M=\mathbb{R}^{n}$.

By convention, the Lévy-Chentsov $\mathrm{S} \alpha \mathrm{S}$ field is pinned down to zero at some point $o \in \mathrm{M}\left(Z_{\alpha}(o)=0\right)$, so $\mu\left(A_{o}\right)=0$. We are in particular interested in those processes that have stationary increments. This notion is well understood in the time series context. To introduce this notion for M-indexed processes, we consider in addition, a group action $G$ on the metric space $\mathrm{M}$, that is, a mapping from $G \times \mathrm{M}$ to $\mathrm{M}$, denoted by $(g, x) \mapsto g x$. We then say that $\left\{Z_{\alpha}(x)\right\}_{x \in \mathrm{M}}$ has stationary increments with respect to $G$, if

$$
\left\{Z_{\alpha}(g(x))-Z_{\alpha}(g(o))\right\}_{x \in \mathrm{M}} \stackrel{d}{=}\left\{Z_{\alpha}(x)\right\}_{x \in \mathrm{M}} \text { for all } g \in G .
$$

In the case $\mathrm{M}=\mathbb{R}$, take $G=\mathbb{R}$ and $g x=x+g$ for all $g, x \in \mathbb{R}$. To verify (2.2), for Gaussian fields, it suffices to verify the covariances. For non-Gaussian stable ones, the above condition can be checked by verifying

$$
\mu\left(\bigcap_{j=1}^{d} A_{g\left(x_{j}\right)}^{\delta_{j}} \Delta A_{g(o)}\right)=\mu\left(\bigcap_{j=1}^{d} A_{x_{j}}^{\delta_{j}}\right),
$$

for all $d \in \mathbb{N}, x_{1}, \ldots, x_{d} \in \mathbf{M}, \boldsymbol{\delta} \in \Lambda^{d}$ (see [35, Theorem 8.2.6]).

Remark 2.1. The pinning-down assumption $Z_{\alpha}(o)=0$ is only a convention. One can easily show that if $Z_{\alpha}$ has stationary increments in the sense of (2.2), then for the process $Z_{\alpha}^{\prime}$ defined by $Z_{\alpha}^{\prime}(x):=Z_{\alpha}(x)+Y, x \in \mathrm{M}$ for any random variable $Y$, not necessarily depending on $Z_{\alpha}$, we have $\left\{Z_{\alpha}^{\prime}(g(x))-Z_{\alpha}^{\prime}(g(o))\right\}_{x \in \mathrm{M}} \stackrel{d}{=}\left\{Z_{\alpha}^{\prime}(x)-\right.$ $\left.Z_{\alpha}^{\prime}(o)\right\}_{x \in \mathrm{M}}$.

2.2. Lévy-Chentsov stable fields. Now, we review the Chentsov representation of Lévy-Chentsov $\mathrm{S} \alpha \mathrm{S}$ fields indexed by a metric space $\mathrm{M}=\mathbb{R}^{n}, \mathbb{S}^{n}, \mathbb{H}^{n}$. by specifying in each case the choice of

$$
(\mathrm{M}, \mathrm{d}), o, G,(E, \mathcal{E}, \mu),\left\{A_{x}\right\}_{x \in \mathrm{M}} \text {. }
$$

Here, for each choice of $(\mathrm{M}, \mathrm{d}), \mathrm{d}$ is the geodesic metric on $\mathrm{M}, o$ is an (arbitrary) fixed starting point where the process is pinned down to zero, $G$ is a group action on $\mathrm{M}$. We need the following assumption.

Assumption 2.2. (i) $(E, \mathcal{E}, \mu)$ and $\left\{A_{x}\right\}_{x \in \mathrm{M}}$ are chosen so that $\mathrm{d}$ is a measure definite kernel associated with $(E, \mathcal{E}, \mu),\left\{A_{x}\right\}_{x \in \mathrm{M}}$ via (1.3). 
(ii) The group action $G$ acts also on $E$ as another group action, such that

$$
A_{g(x)} \Delta A_{g(y)}=g\left(A_{x} \Delta A_{y}\right)=\left(g A_{x}\right) \Delta\left(g A_{y}\right), \quad \text { for all } g \in G, x, y \in \mathrm{M} .
$$

(iii) The measure $\mu$ is $G$-invariant.

(iv) $A_{o}=\emptyset$.

Remark 2.3. Assumptions (ii) (iii) and (iii) imply that $g$ preserves the metric, since

$$
\mathrm{d}(g(x), g(y))=\mu\left(A_{g(x)} \Delta A_{g(y)}\right)=\mu\left(g\left(A_{x} \Delta A_{y}\right)\right)=\mu\left(A_{x} \Delta A_{y}\right)=\mathrm{d}(x, y) .
$$

Remark 2.4. Our presentation is slightly different from [38, 39], where the three cases can be put in a unified framework of projective geometry. See also with [19].

Below are the notations (2.4) in each case. For Euclidean spaces and spheres, more background on group actions can be found in for example [36]. For hyperbolic spaces, see [8] for a review for probabilists.

Example 2.5 (Euclidean space). This is referred to as the Lévy-Chentsov stable fields in 35]. We set $M=\mathbb{R}^{n}, E$ as the space of all hyperplanes of $\mathbb{R}^{n}$ not including the origin, parametrized as $E:=\left\{(s, r): s \in \mathbb{S}^{n}, 0<r<\infty\right\}$, $A_{x}:=\left\{(s, r): s \in \mathbb{S}^{n}, 0<r<\langle s, x\rangle\right\}, x \in \mathbb{R}^{n}$ as the set of all hyperplanes that separate $o$ and $x$, and $\mu(d s d r)=d s d r\left(d s\right.$ is the Lebesgue measure on $\left.\mathbb{S}^{n}\right)$. In this case, $G$ is the rigid body motion on $\mathbb{R}^{n}$, and acts on $E$ in the canonical way.

Example 2.6 (Euclidean sphere). We set $M=\mathbb{S}^{n}:=\left\{x \in \mathbb{R}^{n+1}:\|x\|=1\right\}$, and $E$ is the space of all totally geodesic submanifolds of $\mathbb{S}^{n}$, each denoted by $h_{x}:=$ $\left\{y \in \mathbb{S}^{n}:\langle x, y\rangle=0\right\}, x \in \mathbb{S}^{n}$. Set $A_{x}:=\left\{y \in \mathbb{S}^{n}: h_{y}\right.$ separates $x$ and $\left.o\right\}, x \in \mathbb{S}^{n}, \mu$ is the Lebesgue measure on $\mathbb{S}^{n}$ and $G=S O(n+1)$. The induced action on $E$ is $g h_{x}=h_{g(x)}$.

Remark 2.7. An equivalent and more common representation is as follows: introduce the hemisphere $H_{x}:=\left\{y \in \mathbb{S}^{n}:\langle x, y\rangle>0\right\}$, and define

$$
Z_{\alpha}^{\prime}(x):=\int_{E}\left(\mathbf{1}_{H_{x}}-\mathbf{1}_{H_{o}}\right) d M_{\alpha}, x \in \mathrm{M}
$$

with the same $\mathrm{S} \alpha \mathrm{S}$ random measure. Indeed, one can show that $Z_{\alpha}^{\prime}$ has the same distribution as

$$
\left\{\int_{E}\left|\mathbf{1}_{H_{x}}-\mathbf{1}_{H_{o}}\right| d M_{\alpha}\right\}_{x \in \mathrm{M}} \stackrel{d}{=}\left\{\int_{E} \mathbf{1}_{H_{x} \Delta H_{o}} d M_{\alpha}\right\}_{x \in \mathrm{M}}
$$

by a straightforward calculation, and also $H_{x} \Delta H_{o}=A_{x}$ for all $x \in \mathrm{M}$.

Example 2.8 (Hyperbolic space). For the sake of simplicity, we only describe $\mathbb{H}^{2}$. Consider $\mathrm{M}=\mathbb{D} \equiv\{z \in \mathbb{C}:|z|<1\} \cong \mathbb{H}^{2}$, the Poincaré disc, with

$$
\mathrm{d}\left(z, z^{\prime}\right)=\frac{1}{2} \log \frac{\left|1-\bar{z} z^{\prime}\right|+\left|z-z^{\prime}\right|}{\left|1-\bar{z} z^{\prime}\right|-\left|z-z^{\prime}\right|}, z, z^{\prime} \in \mathbb{D},
$$

and

$$
G \equiv S U(1,1)=\left\{\left(\begin{array}{cc}
\alpha & \beta \\
\bar{\beta} & \bar{\alpha}
\end{array}\right):|\alpha|^{2}-|\beta|^{2}=1, \alpha, \beta \in \mathbb{C}\right\} .
$$

Let $E$ denote the collection of all geodesic lines of $\mathbb{D}$ : each $h \in E$ is an intersection of an Euclidean circle, say $S_{h}$, with $\mathbb{D}$, including the diameters of $\mathbb{D}$ viewed as the intersections of Euclidean circles with infinite diameters. We parameterize $h \in E$ by $(\varphi, \psi) \in[0, \pi / 2] \times[0,2 \pi)$, with $S_{h}=\left\{e^{i(\psi+\varphi)}, e^{i(\psi-\varphi)}\right\}$, 
and take $\mu(d h)=(\sin \varphi)^{-2} c d \varphi d \psi$ (intuitively, $\varphi$ is for the size and $\psi$ for the direction). Then $G$ acts on $E, G^{*}=G$, and $\mu$ is $S U(1,1)$-invariant on $E$. Take $A_{z}:=\{h \in E: h$ separates $z$ and 0$\}, z \in \mathbb{D}$.

Remark 2.9. Strictly speaking, in the case $\alpha=2$, our representations above differ from the corresponding ones in the literature by a multiplicative constant of $\sqrt{2}$. This is easily seen as for our random measure, $M_{2}(A) \sim S_{2}\left(\mu(A)^{1 / 2}, 0,0\right)$ is a centered Gaussian random variable with variance $2 \mu(A)$, while often a Gaussian random measure with control measure $\mu$ evaluated at $A$ is defined to have variance $\mu(A)$.

2.3. Lévy-Chentsov sub-stable fields. There is a simple trick to obtain a new $\mathrm{S} \alpha \mathrm{S}$ field by multiplying to an old $\mathrm{S} \alpha^{\prime} \mathrm{S}$ one (with $\left.\alpha^{\prime} \in(\alpha, 2]\right)$ an independent totally skewed $\alpha / \alpha^{\prime}$-stable random variable, and the so-obtained fields are known as sub-stable fields (or sub-Gaussian when $\alpha^{\prime}=2$ ). Fix $\alpha$ and $\alpha^{\prime}$ so that $\alpha^{\prime} \in$ $(\alpha, 2]$, and let $\xi$ be a totally skewed stable random variable with law determined by $\mathbb{E} e^{-\theta \xi}=e^{-\theta^{\alpha / \alpha^{\prime}}}$ for all $\theta \geq 0$. Then in our context, we refer to

$$
Z_{\alpha, \alpha^{\prime}}(x):=\xi^{1 / \alpha^{\prime}} Z_{\alpha^{\prime}}(x), x \in \mathrm{M},
$$

as a Lévy-Chentsov sub-stable $S \alpha S$ field. It is however not of Chentsov type. The characteristic function of $Z_{\alpha, \alpha^{\prime}}$ is

$$
\mathbb{E} \exp \left(-i \sum_{j=1}^{d} \theta_{j} Z_{\alpha, \alpha^{\prime}}\left(x_{j}\right)\right)=\exp \left(-\left\|\sum_{j=1}^{d} \theta_{j} \mathbf{1}_{A_{x_{j}}}\right\|_{\alpha^{\prime}}^{\alpha}\right) \text {, for all } \boldsymbol{\theta} \in \mathbb{R}^{n},
$$

with $\|\cdot\|_{\alpha^{\prime}}=\int_{E}|\cdot|^{\alpha^{\prime}} d \mu$. See [35, Proposition 3.8.2] for more details. The fact that the right-hand side above is a valid characteristic function has been known since at least [14]. Recently, Istas [18] revisited this fact without making connection to the sub-stable representation (2.6).

\section{A new family of stable processes}

3.1. Set-indexed Karlin stable processes. We now introduce a family of setindexed stable processes, of which our extensions of Lévy Brownian fields are special cases. We shall understand the law of the processes by their finite-dimensional distributions (see Remark 3.3 for issues on their sample paths). Throughout, we assume that $\alpha \in(0,2]$ and $\beta \in(0,1)$.

Let $(E, \mathcal{E})$ be a measure space with a $\sigma$-finite measure $\mu$. Let $\mathcal{A}$ be the family of subsets of $\mathcal{E}$ with finite $\mu$-measure. We let $\mathbb{M}_{p}(E)$ denote the canonical space of Radon point measures on $(E, \mathcal{E})$, equipped with the Borel $\sigma$-algebra $\mathcal{M}_{p}(E)$. In particular, every $m \in \mathbb{M}_{p}(E)$ takes the form $m=\sum_{i \in \mathbb{N}} \delta_{x_{i}}$ with $x_{i} \in E$, and for all $A \in \mathcal{E}, m(A) \in \mathbb{N} \cup\{0, \infty\}$ and $m(A)<\infty$ if $A$ is compact in M. For background on

topological issues, see [30, Chapter 3]. Given $r>0$, let $\mathbb{P}_{r, \mu}^{\prime}$ denote the probability measure on $\left(\mathbb{M}_{p}(E), \mathcal{M}_{p}(E)\right)$ induced by the Poisson point process on $(E, \mathcal{E})$ with mean measure $r \cdot \mu$. That is,

$$
\mathbb{P}_{r, \mu}^{\prime}(m(A)=k)=\frac{(r \mu(A))^{k}}{k !} e^{-r \mu(A)}, k=0,1, \ldots, A \in \mathcal{A}
$$

Set

$$
\mu_{\beta}(\cdot):=c_{\beta} \int_{0}^{\infty} r^{-\beta-1} \mathbb{P}_{r, \mu}^{\prime}(\cdot) d r \quad \text { with } \quad c_{\beta}:=\frac{\beta 2^{1-\beta}}{\Gamma(1-\beta)}
$$


as a $\sigma$-finite measure on $\left(\mathbb{M}_{p}(E), \mathcal{M}_{p}(E)\right)$. We introduce the set-indexed Karlin stable process given by

$$
Y_{\alpha, \beta}(A):=\int_{\mathbb{M}_{p}(E)} \mathbf{1}_{\{m(A) \text { odd }\}} M_{\alpha, \beta}(d m), A \in \mathcal{A},
$$

where $M_{\alpha, \beta}$ is an $\mathrm{S} \alpha \mathrm{S}$ random measure on $\mathbb{M}_{p}$ with control measure $\mu_{\beta}$. Note that the process $Y$ is still of Chentsov type: by introducing

$$
A^{*}:=\left\{m \in \mathbb{M}_{p}(E): m(A) \text { odd }\right\}, A \in \mathcal{A},
$$

we have

$$
Y_{\alpha, \beta}(A)=\int_{\mathbb{M}_{p}(E)} \mathbf{1}_{A^{*}} M_{\alpha, \beta}(d m) .
$$

Remark 3.1. The original Karlin stable processes investigated in [9] corresponds to

$$
Y_{\alpha, \beta}(t) \equiv Y_{\alpha, \beta}([0, t])=\int_{\mathbb{M}_{p}\left(\mathbb{R}_{+}\right)} \mathbf{1}_{\{m([0, t]) \text { odd }\}} M_{\alpha, \beta}(d m)
$$

which has a more convenient representation

$$
\left\{Y_{\alpha, \beta}(t)\right\}_{t \in \mathbb{R}_{+}} \stackrel{d}{=}\left\{\int_{\mathbb{R}_{+} \times \Omega^{\prime}} \mathbf{1}_{\left\{N^{\prime}(r t) \text { odd }\right\}} \widetilde{M}_{\alpha, \beta}\left(d r d \omega^{\prime}\right)\right\}_{t \in \mathbb{R}_{+}},
$$

where $N^{\prime}$ is a standard Poisson point process on $\mathbb{R}_{+}$, defined on another probability space $\left(\Omega^{\prime}, \mathcal{F}^{\prime}, \mathbb{P}^{\prime}\right)$ and $\widetilde{M}_{\alpha, \beta}$ is an S $\alpha \mathrm{S}$ random measure on $\mathbb{R}_{+} \times \Omega^{\prime}$ with control measure $c_{\beta} r^{-\beta-1} d r \mathbb{P}^{\prime}\left(d \omega^{\prime}\right)$. However, such a representation cannot be extended to the case $\mathrm{M}=\mathbb{S}^{n}$ or $\mathbb{H}^{n}$ (e.g. in the case of $\mathbb{S}^{n}$ the 'scaled set' $r A_{x}$ would not make sense). Instead we work with stochastic integrals over $\mathbb{M}_{p}(E)$.

Remark 3.2. Another representation of (3.2) with a flavor of doubly stochastic processes [35] is to write

$$
\left\{Y_{\alpha, \beta}(A)\right\}_{t \in \mathcal{A}} \stackrel{d}{=}\left\{\int_{\mathbb{R}_{+} \times \Omega^{\prime}} \mathbf{1}_{\left\{N_{r}^{\prime}(A) \text { odd }\right\}} \widetilde{M}_{\alpha, \beta}^{\prime}\left(d r d \omega^{\prime}\right)\right\}_{t \in \mathbb{R}_{+}},
$$

where $\left\{N_{r}^{\prime}\right\}_{r>0}$ is a family of independent Poisson point processes on $(E, \mathcal{E})$ with intensity measure $r \mu$ respectively, defined on another probability space $\left(\Omega^{\prime}, \mathcal{F}^{\prime}, \mathbb{P}^{\prime}\right)$.

Remark 3.3. For the Karlin stable process in (3.3), in the Gaussian case it is a fractional Brownian motion with Hurst index $H=\beta / 2 \in(0,1)$, and hence the path properties are well known. We expect then to be able to improve and obtain regularity results on sample paths (see [21] for $\mathrm{M}=\mathbb{S}^{n}$ ). In the stable case, however, even for $\mathrm{M}=\mathbb{R}_{+}$it remains open whether the Karlin stable process (3.3) has a version in the space $D$ for $\alpha \in[1,2)$ (see $\underline{9}$, Remark 2]).

3.2. Fractional Lévy-Chentsov stable fields. For a metric space (M,d) along with notations (2.4) satisfying Assumption 2.2, we name the process

$$
\eta_{\alpha, \beta}(x):=Y_{\alpha, \beta}\left(A_{x}\right), x \in \mathrm{M}
$$

as the fractional Lévy-Chentsov stable field with parameters $\alpha \in(0,2], \beta \in(0,1)$. Our main result is the following.

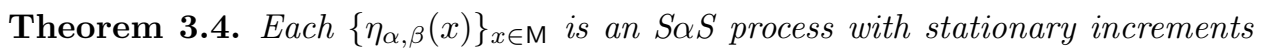
as in (2.2). 
Proof. It is equivalent to prove, for all $g \in G$,

$$
\left\{\int_{\mathbb{M}_{p}(E)} \mathbf{1}_{A_{g(x)}^{*} \Delta A_{g(o)}^{*}} M_{\alpha, \beta}(d m)\right\}_{x \in \mathrm{M}} \stackrel{d}{=}\left\{\int_{\mathbb{M}_{p}(E)} \mathbf{1}_{A_{x}^{*}} M_{\alpha, \beta}(d m)\right\}_{x \in \mathrm{M}} .
$$

The above is equivalent to (recall (2.3) $)$, for all $d \in \mathbb{N}, \boldsymbol{\delta} \in \Lambda^{d}, x_{1}, \ldots, x_{d} \in \mathrm{M}$,

$$
\mu_{\beta}\left(\bigcap_{j=1}^{d}\left(A_{g\left(x_{j}\right)}^{*}\right)^{\delta_{j}} \Delta A_{g(o)}^{*}\right)=\mu_{\beta}\left(\bigcap_{j=1}^{d}\left(A_{x_{j}}^{*}\right)^{\delta_{j}}\right) .
$$

We have

$$
\begin{aligned}
A_{g(x)}^{*} \Delta A_{g(y)}^{*} & =\left\{m\left(A_{g(x)}\right) \text { odd }\right\} \Delta\left\{m\left(A_{g(y)}\right) \text { odd }\right\}=\left\{m\left(A_{g(x)} \Delta A_{g(y)}\right) \text { odd }\right\} \\
& =\left\{m\left(\left(g A_{x}\right) \Delta\left(g A_{y}\right)\right) \text { odd }\right\}=\left(g A_{x}\right)^{*} \Delta\left(g A_{y}\right)^{*},
\end{aligned}
$$

where in the third step we applied (2.5), and

$$
\left(A_{g(x)}^{*}\right)^{c} \Delta A_{g(y)}^{*}=\left(A_{g(x)}^{*} \Delta A_{g(y)}^{*}\right)^{c}=\left(\left(g A_{x}\right)^{*} \Delta\left(g A_{y}\right)^{*}\right)^{c}=\left(g A_{x}\right)^{* c} \Delta\left(g A_{y}\right)^{*} .
$$

Then, (3.5) becomes (note $g A_{o}=\emptyset$ )

$$
\mu_{\beta}\left(\bigcap_{j=1}^{d}\left(g A_{x_{j}}\right)^{* \delta_{j}}\right)=\mu_{\beta}\left(\bigcap_{j=1}^{d}\left(A_{x_{j}}^{*}\right)^{\delta_{j}}\right) .
$$

The above shall follow from (recall $\mu_{\beta}$ in (3.1)), for all $r>0$,

$$
\begin{aligned}
\mathbb{P}_{r, \mu}^{\prime}\left(m\left(g A_{x_{j}}\right)=\delta_{j} \quad \bmod 2, j\right. & =1, \ldots, d) \\
& =\mathbb{P}_{r, \mu}^{\prime}\left(m\left(A_{x_{j}}\right)=\delta_{j} \quad \bmod 2, j=1, \ldots, d\right) .
\end{aligned}
$$

The left-hand side above can be expressed as

$$
\widetilde{\mathbb{P}}_{r, \mu}^{\prime}\left(m\left(A_{x_{j}}\right)=\delta_{j} \quad \bmod 2, j=1, \ldots, d\right),
$$

where $\widetilde{\mathbb{P}}_{r, \mu}^{\prime}$ is the probability measure on $\mathbb{M}_{p}(E)$ induced by the Poisson point process on $E$ with intensity measure $r \cdot \mu(g \cdot)$. Since $\mu$ is $G$-invariant, the probability above is nothing but the right-hand side of (3.6). This completes the proof.

The law of the increment of the field over any two points is uniquely determined by their geodesic distance.

Proposition 3.5. For the stable process $\eta_{\alpha, \beta}$ defined in (3.4), we have that

$$
\frac{\eta_{\alpha, \beta}(x)-\eta_{\alpha, \beta}(y)}{\mathrm{d}^{\beta / \alpha}(x, y)} \sim S_{\alpha}(1,0,0), \text { for all } x, y \in \mathrm{M} .
$$

Proof. This follows from a straightforward computation:

$$
\begin{aligned}
\mathbb{E} \exp \left(i \theta\left(\eta_{\alpha, \beta}(x)-\eta_{\alpha, \beta}(y)\right)\right) & =\exp \left(-|\theta|^{\alpha} \int_{\mathbb{M}_{p}(E)} \mathbf{1}_{\left\{m\left(A_{x} \Delta A_{y}\right) \text { odd }\right\}} \mu_{\beta}(d m)\right) \\
& =\exp \left(-\mu_{\beta}\left(A_{x}^{*} \Delta A_{y}^{*}\right)|\theta|^{\alpha}\right)=\exp \left(-\mathrm{d}^{\beta}(x, y)|\theta|^{\alpha}\right) .
\end{aligned}
$$

In the last step, we applied an identity regarding $\mathrm{d}^{\beta}$ and $\mu_{\beta}$, established separately in Proposition 4.1 
Remark 3.6. The Karlin stable fields indexed by metric spaces $\eta_{\alpha, \beta} \equiv$ $\left\{\eta_{\alpha, \beta}(x)\right\}_{x \in \mathrm{M}}$ are different from Lévy-Chentsov stable fields and Lévy-Chentsov sub-stable fields. To see this, it suffices to compare the spherical representations of finite-dimensional distributions 35]. The Lévy-Chentsov sub-stable fields are spectrally continuous, while the other two are spectrally discrete, of which one could check readily that the spectral measures are different. In the case $M=\mathbb{R}^{n}$, it is also easily seen to be different from the spectrally discrete Takenaka random fields 37].

We conclude this section with a few immediate consequences on the so-called setindexed fractional Brownian motions investigated by Herbin and Merzbach [15, 16], whose motivation is different from ours.

3.3. Set-indexed fractional Brownian motions. Recall the set-indexed Karlin stable process $\left\{Y_{\alpha, \beta}(A)\right\}_{A \in \mathcal{A}}$ as in (3.2). In the Gaussian case, one could compute

$$
\operatorname{Cov}\left(Y_{2, \beta}(A), Y_{2, \beta}(B)\right)=\mu^{\beta}(A)+\mu^{\beta}(B)-\mu^{\beta}(A \Delta B), A, B \in \mathcal{A},
$$

and recognize the covariance structure of set-indexed fractional Brownian motions (the above actually differs from the one in [15, 16] by a multiplicative constant of $1 / 2$; see a detailed calculation below). A special choice of the index set is the collection of rectangles in Euclidean space

$$
[\mathbf{0}, \boldsymbol{t}] \equiv\left\{\boldsymbol{s}=\left(s_{1}, \ldots, s_{n}\right): 0 \leq s_{j} \leq t_{j}, j=1, \ldots, n\right\}, \boldsymbol{t}=\left(t_{1}, \ldots, t_{n}\right) \in \mathbb{R}_{+}^{n},
$$

in which case the process is referred to as a multiparameter fractional Brownian motion (see also 31, 32]). This process has different properties from other extensions of fractional Brownian motions, and in particular it does not have stationary increments (see e.g. 31] for comparisons). Following the same notion, we refer to

$$
\xi_{\alpha, \beta}(\boldsymbol{t}):=Y_{\alpha, \beta}(\boldsymbol{t}) \equiv \int_{\mathbb{M}_{p}(E)} \mathbf{1}_{\{m([\mathbf{0}, \boldsymbol{t}]) \text { odd }\}} M_{\alpha, \beta}(d m), \boldsymbol{t} \in \mathbb{R}_{+}^{n}
$$

as a multiparameter fractional stable field. This integral representation in the Gaussian case $(\alpha=2)$ seems new.

We next consider a natural decomposition of set-indexed fractional Brownian motions $Y_{2, \beta}$, inspired by the decomposition of fractional Brownian motions by bifractional Brownian motions introduced by Lei and Nualart [23]. Write $W_{\beta} \equiv Y_{2, \beta}$ from now on. We consider a slightly different representation of $W_{\beta}$ :

$$
\left\{W_{\beta}(A)\right\}_{A \in \mathcal{A}} \stackrel{d}{=}\left\{\int_{\mathbb{R}_{+} \times \mathbb{M}_{p}(E)} \mathbf{1}_{\{m(A) \text { odd }\}} \widetilde{M}_{2, \beta}(d r d m)\right\}_{A \in \mathcal{A}},
$$

where $\widetilde{M}_{2, \beta}$ is a Gaussian (S $\alpha$ S with $\left.\alpha=2\right)$ random measure on $\mathbb{R}_{+} \times \mathbb{M}_{p}(E)$ with control measure $c_{\beta} r^{-\beta-1} d r \mathbb{P}_{r, \mu}^{\prime}(d m)$, and then consider its decomposition

with

$$
\left\{W_{\beta}(A)\right\}_{A \in \mathcal{A}} \stackrel{d}{=}\left\{W_{1, \beta}(A)+W_{2, \beta}(A)\right\}_{A \in \mathcal{A}}
$$

$$
\begin{aligned}
W_{1, \beta}(A) & :=\int_{\mathbb{R}_{+} \times \mathbb{M}_{p}(E)}\left(\mathbf{1}_{\{m(A) \text { odd }\}}-q_{r}(A)\right) \widetilde{M}_{2, \beta}(d r d m) \\
W_{2, \beta}(A) & :=\int_{\mathbb{R}_{+} \times \mathbb{M}_{p}(E)} q_{r}(A) \widetilde{M}_{2, \beta}(d r d m) \\
q_{r}(A) & :=\mathbb{P}_{r, \mu}^{\prime}(m(A) \text { odd }) .
\end{aligned}
$$


For the case $\mathrm{M}=\mathbb{R}_{+}$and relation to decomposition of fractional Brownian motions, see [10, Section 2.2].

Proposition 3.7. $\left\{W_{1, \beta}(A)\right\}_{A \in \mathcal{A}}$ and $\left\{W_{2, \beta}(A)\right\}_{A \in \mathcal{A}}$ are independent centered Gaussian processes with covariance functions, for $A_{1}, A_{2} \in \mathcal{A}$,

$$
\begin{aligned}
& \operatorname{Cov}\left(W_{1, \beta}\left(A_{1}\right), W_{1, \beta}\left(A_{2}\right)\right)=\left(\mu\left(A_{1}\right)+\mu\left(A_{2}\right)\right)^{\beta}-\mu^{\beta}\left(A_{1} \Delta A_{2}\right) \\
& \operatorname{Cov}\left(W_{2, \beta}\left(A_{1}\right), W_{2, \beta}\left(A_{2}\right)\right)=\mu^{\beta}\left(A_{1}\right)+\mu^{\beta}\left(A_{2}\right)-\left(\mu\left(A_{1}\right)+\mu\left(A_{2}\right)\right)^{\beta} .
\end{aligned}
$$

Proof. Recall that for a Poisson random variable $Z$ with parameter $\lambda>0$,

$$
\mathbb{P}(Z \text { is odd })=\frac{1}{2}\left(1-e^{-2 \lambda}\right) \text {. }
$$

We first compute the covariance function of $\left\{W_{2, \beta}(A)\right\}_{A \in \mathcal{A}}$. For $A_{1}, A_{2} \in \mathcal{A}$,

$$
\begin{aligned}
\operatorname{Cov}\left(W_{2, \beta}\left(A_{1}\right), W_{2, \beta}\left(A_{2}\right)\right) & =2 \int_{\mathbb{R}_{+}} q_{r}\left(A_{1}\right) q_{r}\left(A_{2}\right) c_{\beta} r^{-\beta-1} d r \\
& =2 \int_{\mathbb{R}_{+}} \frac{1}{4}\left(1-e^{-2 r \mu\left(A_{1}\right)}\right)\left(1-e^{-2 r \mu\left(A_{2}\right)}\right) c_{\beta} r^{-\beta-1} d r
\end{aligned}
$$

(the factor 2 is due to our convention for the Gaussian random measure, see Remark [2.9), and for $\beta \in(0,1)$, the desired formula then follows immediately from the identity for the Gamma function

$$
\int_{0}^{\infty}\left(1-e^{-a r}\right) r^{-\beta-1} d r=\frac{a^{\beta} \Gamma(1-\beta)}{\beta}, a>0 .
$$

Similarly,

$$
\begin{aligned}
& \operatorname{Cov}\left(W_{1, \beta}\left(A_{1}\right), W_{1, \beta}\left(A_{2}\right)\right) \\
& \quad=2 \int_{\mathbb{R}_{+} \times \mathbb{M}_{p}(E)}\left(\mathbb{P}_{r, \mu}^{\prime}\left(m\left(A_{1}\right) \text { odd, } m\left(A_{2}\right) \text { odd }\right)-q_{r}\left(A_{1}\right) q_{r}\left(A_{2}\right)\right) c_{\beta} r^{-\beta-1} d r,
\end{aligned}
$$

and

$$
\mathbb{P}_{r, \mu}^{\prime}\left(m\left(A_{1}\right) \text { odd, } m\left(A_{2}\right) \text { odd }\right)=\frac{1}{4}\left(1-e^{-2 r \mu\left(A_{1}\right)}-e^{-2 r \mu\left(A_{2}\right)}+e^{-2 r \mu\left(A_{1} \Delta A_{2}\right)}\right) .
$$

The desired covariance formula then follows. The independence of $\left\{W_{1, \beta}(A)\right\}_{A \in \mathcal{A}}$ and $\left\{W_{2, \beta}(A)\right\}_{A \in \mathcal{A}}$ can be verified similarly.

\section{Measure definite Kernels}

In this section, we extract a result on measure definite kernels that we developed and used implicitly in our previous analysis. This result is of independent interest for metric analysis.

Let $(\mathrm{M}, \mathrm{d})$ be a metric space. Recall that the metric $\mathrm{d}$ is of conditionally negative type, if for all $n \geq 2$ and $x_{1}, \ldots, x_{n} \in \mathrm{M}$ and $\lambda_{1}, \ldots, \lambda_{n} \in \mathbb{R}$ with $\sum_{i=1}^{n} \lambda_{i}=0$, we have $\sum_{i=1}^{n} \sum_{j=1}^{n} \lambda_{i} \lambda_{j} \mathrm{~d}\left(x_{i}, x_{j}\right) \leq 0$. Recall the definition of a metric $\mathrm{d}$ being a measure definite kernel in (1.3). Sometimes it is convenient to write equivalently

$$
\mathrm{d}(x, y)=\int\left|\mathbf{1}_{A_{x}}-\mathbf{1}_{A_{y}}\right| d \mu, \text { for all } x, y \in \mathrm{M} .
$$


A measure definite kernel as a metric is necessarily of conditionally negative type, as for all collections $\left\{A_{i}\right\}_{i=1, \ldots, n} \subset\left\{A_{x}\right\}_{x \in \mathrm{M}}$,

$$
\left(\sum_{i=1}^{n} \lambda_{i} \mathbf{1}_{A_{i}}\right)^{2}=\frac{1}{2} \sum_{i=1}^{n} \sum_{j=1}^{n} \lambda_{i} \lambda_{j}\left(\mathbf{1}_{A_{i}}+\mathbf{1}_{A_{j}}-\mathbf{1}_{A_{i} \Delta A_{j}}\right)=-\frac{1}{2} \sum_{i=1}^{n} \sum_{j=1}^{n} \lambda_{i} \lambda_{j} \mathbf{1}_{A_{i} \Delta A_{j}} .
$$

Although the converse is not true. It is well known that the mapping $d \mapsto d^{\beta}$ preserves the property of being conditionally negative. The following shows that the mapping also preserves the property of being a measure definite kernel. Recall notations for $\mathbb{M}_{p}(E), \mathcal{M}_{p}(E), \mu_{\beta}, \mathbb{P}_{r, \mu}^{\prime}, A^{*}$ as in Section 3.1

Proposition 4.1. Suppose that $(M, \mathrm{~d})$ is a metric space and $\mathrm{d}$ is a measure definite kernel with respect to a measure space $(E, \mathcal{E}, \mu)$ and $\left\{A_{x}\right\}_{x \in \mathrm{M}} \subset \mathcal{E}$, as in (1.3). Then for all $\beta \in(0,1)$,

$$
\mathrm{d}^{\beta}(x, y)=\mu_{\beta}\left(A_{x}^{*} \Delta A_{y}^{*}\right),
$$

with $\mu_{\beta}$ as in (3.1) and $A^{*}:=\left\{m \in \mathbb{M}_{p}(E): m(A)\right.$ odd $\}, A \in \mathcal{A}$.

Proof. First, since $\mu\left(A_{x} \Delta A_{y}\right)=\mathrm{d}(x, y)$, by (3.8) we write

$$
\mathrm{d}^{\beta}(x, y)=\int_{\mathbb{R}_{+}} \frac{1}{2}\left(1-e^{-2 r \mu\left(A_{x} \Delta A_{y}\right)}\right) c_{\beta} r^{-\beta-1} d r .
$$

On the other hand, for every $r>0$, recalling (3.7), we have

$$
\begin{aligned}
\mathrm{d}^{\beta}(x, y) & =\int_{\mathbb{R}_{+}} \mathbb{P}_{r, \mu}^{\prime}\left(\left\{m\left(A_{x}\right) \text { odd }\right\} \Delta\left\{m\left(A_{y}\right) \text { odd }\right\}\right) c_{\beta} r^{-\beta-1} d r \\
& =\int_{\mathbb{R}_{+}} \int_{\mathbb{M}_{p}(E)}\left|\mathbf{1}_{\left\{m\left(A_{x}\right) \text { odd }\right\}}-\mathbf{1}_{\left\{m\left(A_{y}\right) \text { odd }\right\}}\right| \mathbb{P}_{r, \mu}^{\prime}(d m) c_{\beta} r^{-\beta-1} d r \\
& =\int_{\mathbb{M}_{p}(E)}\left|\mathbf{1}_{A_{x}^{*}}-\mathbf{1}_{A_{y}^{*}}\right| \mu_{\beta}(d m)=\mu_{\beta}\left(A_{x}^{*} \Delta A_{y}^{*}\right) .
\end{aligned}
$$

\section{A Limit TheOREM FOR SET-INDEXed Karlin StABle PROCESSES}

Limit theorems for stochastic processes indexed by metric spaces are not new (e.g. [4, [5, 22, 28]). However, very few theorems have been known beside $\mathbb{R}^{n}$ and $\mathbb{R}_{+}^{n}$-indexed examples, with the notable exception for sphere-indexed processes investigated by Estrade and Istas [11]. Our model is a variation of an infinite urn scheme considered by Karlin [20]. This version of the model was introduced for $\mathrm{M}=\mathbb{R}_{+}$in the proofs of [9] as the Poissonized version of the corresponding Karlin model, and has an aggregation nature.

Let $(E, \mathcal{E}, \mu)$ be a measure space with $\mu$ a $\sigma$-finite measure. Let $\left\{p_{k}\right\}_{k \in \mathbb{N}}$ be prescribed strictly positive numbers, and $\rho>0$ a scaling parameter that eventually goes to infinity. For each $\rho>0$, let $\left\{N_{k}^{(\rho)}\right\}_{k \in \mathbb{N}}$ be a family of independent Poisson point processes with mean measure $\rho p_{k} \cdot \mu$ on $(E, \mathcal{E})$ and let $\left\{\varepsilon_{k}\right\}_{k \in \mathbb{N}}$ be another family of i.i.d. random variables, independent from the Poisson point processes. We shall restrict to those sets in $\mathcal{E}$ with finite $\mu$-measure, the collection of which denoted by $\mathcal{A}$. Our model is then defined as

$$
U_{\rho}(A):=\sum_{k \in \mathbb{N}} \varepsilon_{k} \mathbf{1}_{\left\{N_{k}^{(\rho)}(A) \text { odd }\right\}}, \rho>0, A \in \mathcal{A} .
$$


We assume, with $\nu:=\sum_{k \in \mathbb{N}} \delta_{1 / p_{k}}$, that for some $\beta \in(0,1)$,

$$
\nu(t)=\max \left\{k \in \mathbb{N}: p_{k} \geq 1 / t\right\}=t^{\beta} L(t), \quad t>0,
$$

where $L$ is a slowly varying function at $\infty$, and the characteristic function $\phi(\theta)=$ $\mathbb{E} \exp \left(i \theta \varepsilon_{1}\right)$ satisfies

$$
1-\phi(\theta) \sim \sigma_{\varepsilon}^{\alpha}|\theta|^{\alpha} \text { as } \theta \rightarrow 0 .
$$

The above follows for example if for some $\alpha \in(0,2)$,

$$
\lim _{x \rightarrow \infty} \frac{\mathbb{P}\left(\left|\varepsilon_{1}\right|>x\right)}{x^{-\alpha}}=C_{\varepsilon} \in(0, \infty),
$$

and $\sigma_{\varepsilon}^{\alpha}=C_{\varepsilon} \int_{0}^{\infty} x^{-\alpha} \sin x d x$ (e.g. [6, Theorem 8.1.10]), or if $\alpha=2$, $\varepsilon_{1}$ is centered and $\sigma_{\varepsilon}^{2}=\mathbb{E} \varepsilon_{1}^{2} / 2<\infty$. Recall $c_{\beta}$ in (3.1).

Theorem 5.1. Under assumptions (5.1) and (5.2),

$$
\left(\frac{U_{\rho}(A)}{b_{\rho}}\right)_{A \in \mathcal{A}} \stackrel{\text { f.d.d. }}{\Longrightarrow}\left(Y_{\alpha, \beta}(A)\right)_{A \in \mathcal{A}} \quad \text { with } \quad b_{\rho}:=\left(\frac{\beta}{c_{\beta}} \rho^{\beta} L(\rho)\right)^{1 / \alpha} \sigma_{\varepsilon} .
$$

Proof. Consider $d \in \mathbb{N}, \boldsymbol{A}=\left(A_{1}, \ldots, A_{d}\right) \in \mathcal{A}^{d}$ and $\boldsymbol{\delta}=\left(\delta_{1}, \ldots, \delta_{d}\right) \in \Lambda_{d}$, and for any point measure $m$ on $(E, \mathcal{E})$, for the sake of simplicity write

$$
\{\boldsymbol{m}(\boldsymbol{A})=\boldsymbol{\delta} \bmod 2\} \equiv\left\{m\left(A_{j}\right)=\delta_{j} \bmod 2 \text { for all } j=1, \ldots, d\right\} .
$$

The following statistics play a crucial role:

$$
M_{\rho}^{\delta}(A):=\sum_{k \in \mathbb{N}} 1_{\left\{N_{k}^{(\rho)}(A)=\delta \bmod 2\right\}},
$$

Let $N^{(r)}$ be a Poisson point process on $(E, \mathcal{E})$ with intensity measure $r \mu(\cdot)$. Introduce

$$
\begin{aligned}
\mathfrak{m}^{\boldsymbol{\delta}}(\boldsymbol{A}) & :=\int_{\mathbb{R}_{+}} \mathbb{P}\left(\boldsymbol{N}^{(r)}(\boldsymbol{A})=\boldsymbol{\delta} \bmod 2\right) c_{\beta} r^{-\beta-1} d r \\
& =\int_{\mathbb{M}_{p}(E)} \mathbf{1}_{\{\boldsymbol{m}(\boldsymbol{A})=\boldsymbol{\delta} \bmod 2\}} \mu_{\beta}(d m) .
\end{aligned}
$$

The key estimate is

$$
\lim _{\rho \rightarrow \infty} \frac{M_{\rho}^{\delta}(\boldsymbol{A})}{b_{\rho}^{\alpha}}=\frac{\mathfrak{m}^{\delta}(\boldsymbol{A})}{\sigma_{\varepsilon}^{\alpha}} \text { in probability. }
$$

Observe that

$$
\mathbb{E} M_{\rho}^{\boldsymbol{\delta}}(\boldsymbol{A})=\sum_{k \in \mathbb{N}} \mathbb{P}\left(\boldsymbol{N}_{k}^{(\rho)}(\boldsymbol{A})=\boldsymbol{\delta} \bmod 2\right)=\int_{0}^{\infty} \mathbb{P}\left(\boldsymbol{N}^{(\rho / x)}(\boldsymbol{A})=\boldsymbol{\delta} \bmod 2\right) \nu(d x) .
$$

Set $\varphi_{\boldsymbol{A}}(s):=\mathbb{P}\left(\boldsymbol{N}^{(s)}(\boldsymbol{A})=\boldsymbol{\delta} \bmod 2\right)$. It is easy to see that $\varphi_{\boldsymbol{A}}$ is differentiable and vanishes at zero. Therefore, integrating by parts, we have

$$
\mathbb{E} M_{\rho}^{\delta}(\boldsymbol{A})=\int_{0}^{\infty} \varphi_{\boldsymbol{A}}(\rho / x) \nu(d x)=\int_{0}^{\infty} \frac{1}{x^{2}} \varphi_{\boldsymbol{A}}^{\prime}\left(\frac{1}{x}\right) \nu(\rho x) d x
$$


Then,

$$
\begin{aligned}
\lim _{\rho \rightarrow \infty} \frac{\mathbb{E} M_{\rho}^{\boldsymbol{\delta}}(\boldsymbol{A})}{\rho^{\beta} L(\rho)} & =\lim _{\rho \rightarrow \infty} \int_{0}^{\infty} \frac{1}{x^{2}} \varphi_{\boldsymbol{A}}^{\prime}\left(\frac{1}{x}\right) \frac{\nu(\rho x)}{\rho^{\beta} L(\rho)} d x \\
& =\int_{0}^{\infty} \varphi_{\boldsymbol{A}}^{\prime}\left(\frac{1}{x}\right) x^{\beta-2} d x=\int_{0}^{\infty} \varphi_{\boldsymbol{A}}\left(\frac{1}{x}\right) \beta x^{\beta-1} d x \\
& =\int_{0}^{\infty} \mathbb{P}\left(\boldsymbol{N}^{(r)}(\boldsymbol{A})=\boldsymbol{\delta} \bmod 2\right) \beta r^{-\beta-1} d r
\end{aligned}
$$

where in the second step, we applied the assumption (5.1) on $\nu$, and the interchange of the limit and the integral can be verified as explained in [9, Lemma 1]. Furthermore, since $\operatorname{Var}\left(M_{\rho}^{\boldsymbol{\delta}}(\boldsymbol{A})\right) \leq \sum_{k \in \mathbb{N}} \mathbb{P}\left(\boldsymbol{N}_{k}^{(\rho)}(\boldsymbol{A})=\boldsymbol{\delta} \bmod 2\right)=\mathbb{E} M_{\rho}^{\boldsymbol{\delta}}(\boldsymbol{A})$, the $L^{2}$ convergence holds. We have thus shown (5.3).

Now we prove the desired convergence by computing the characteristic function of the finite-dimensional distribution. For all $\boldsymbol{\theta} \in \mathbb{R}^{d}$, we write

$$
\begin{aligned}
\mathbb{E} \exp \left(i \sum_{j=1}^{d} \theta_{j} \frac{U_{\rho}\left(A_{j}\right)}{b_{\rho}}\right) & =\mathbb{E} \exp \left(i \sum_{j=1}^{d} \frac{\theta_{j}}{b_{\rho}} \sum_{k \in \mathbb{N}} \varepsilon_{k} \mathbf{1}_{\left\{N_{k}^{(\rho)}\left(A_{j}\right) \text { odd }\right\}}\right) \\
& =\mathbb{E} \exp \left(i \sum_{\boldsymbol{\delta} \in \Lambda_{d}} \frac{|\langle\boldsymbol{\theta}, \boldsymbol{\delta}\rangle|}{b_{\rho}} \sum_{k \in \mathbb{N}} \varepsilon_{k} \mathbf{1}_{\left\{\boldsymbol{N}_{k}^{(\rho)}(\boldsymbol{A})=\boldsymbol{\delta} \bmod 2\right\}}\right) .
\end{aligned}
$$

Let $\mathcal{N}$ denote the $\sigma$-algebra generated by $\left\{N_{k}\right\}_{k \in \mathbb{N}}$. Recall that $\phi$ is the characteristic function of $\varepsilon_{1}$. Then, the above expression becomes

$$
\mathbb{E}\left\{\prod_{\boldsymbol{\delta} \in \Lambda_{d}} \mathbb{E}\left[\exp \left(i \frac{|\langle\boldsymbol{\theta}, \boldsymbol{\delta}\rangle|}{b_{\rho}} \sum_{\ell=1}^{M_{\rho}^{\boldsymbol{\delta}}(\boldsymbol{A})} \varepsilon_{\ell}^{\boldsymbol{\delta}}\right) \mid \mathcal{N}\right]\right\}=\mathbb{E}\left(\prod_{\boldsymbol{\delta} \in \Lambda_{d}} \phi\left(\frac{|\langle\boldsymbol{\theta}, \boldsymbol{\delta}\rangle|}{b_{\rho}}\right)^{M_{\rho}^{\delta}(\boldsymbol{A})}\right) .
$$

Therefore

$$
\begin{aligned}
\mathbb{E} \exp \left(i \sum_{j=1}^{d} \theta_{j} \frac{U_{\rho}\left(A_{j}\right)}{b_{\rho}}\right) & =\mathbb{E} \exp \left(\sum_{\boldsymbol{\delta} \in \Lambda_{d}} M_{\rho}^{\boldsymbol{\delta}}(\boldsymbol{A}) \log \phi\left(\frac{|\langle\boldsymbol{\theta}, \boldsymbol{\delta}\rangle|}{b_{\rho}}\right)\right) \\
& =\mathbb{E} \exp \left(-\sum_{\boldsymbol{\delta} \in \Lambda_{d}} \frac{M_{\rho}^{\boldsymbol{\delta}}(\boldsymbol{A})}{b_{\rho}^{\alpha}}|\langle\boldsymbol{\theta}, \boldsymbol{\delta}\rangle|^{\alpha} \sigma_{\varepsilon}^{\alpha} \frac{\log \phi\left(\frac{|\langle\boldsymbol{\theta}, \boldsymbol{\delta}\rangle|}{b_{\rho}}\right)}{-\sigma_{\varepsilon}^{\alpha}\left|\frac{\langle\boldsymbol{\theta}, \boldsymbol{\delta}\rangle}{b_{\rho}}\right|^{\alpha}}\right) .
\end{aligned}
$$

Recall that the assumption (5.2) implies that $\log \phi(\theta) \sim \phi(\theta)-1 \sim-\sigma_{\varepsilon}^{\alpha}|\theta|^{\alpha}$ as $\theta \rightarrow$ 0 . Then, by (5.3) and the dominated convergence theorem,

$$
\lim _{\rho \rightarrow \infty} \mathbb{E} \exp \left(i \sum_{j=1}^{d} \theta_{j} \frac{U_{\rho}\left(A_{j}\right)}{b_{\rho}}\right)=\exp \left(-\sum_{\boldsymbol{\delta} \in \Lambda_{d}}|\langle\boldsymbol{\theta}, \boldsymbol{\delta}\rangle|^{\alpha} \mathfrak{m}^{\boldsymbol{\delta}}(\boldsymbol{A})\right),
$$

where the limit is the desired characteristic function $\mathbb{E} \exp \left(i \sum_{j=1}^{d} \theta_{j} Y_{\alpha, \beta}\left(A_{j}\right)\right)$ (recall (2.1)). This completes the proof.

Acknowledgement. The authors would like to thank an anonymous referee for careful reading of the paper. YW thanks Olivier Durieu and Ilya Molchanov for careful reading and inspiring discussions on an earlier draft of the paper. ZF and YW's research were partially supported by Army Research Laboratory grant 
W911NF-17-1-0006. YW's research was in addition partially supported by NSA grant H98230-16-1-0322.

\section{REFERENCES}

[1] Adler, R. J. (1990). An introduction to continuity, extrema, and related topics for general Gaussian processes. Institute of Mathematical Statistics Lecture Notes-Monograph Series, 12. Institute of Mathematical Statistics, Hayward, CA. [2] Adler, R. J. and Taylor, J. E. (2007). Random fields and geometry. Springer Monographs in Mathematics. Springer, New York.

[3] Biermé, H. (2017). Introduction to random fields and scale invariance. To appear in a volume of the CEMPI Lecture Notes in Mathematics subseries. https://hal.archives-ouvertes.fr/hal-01493834v2.

[4] Biermé, H. and Durieu, O. (2014). Invariance principles for self-similar setindexed random fields. Trans. Amer. Math. Soc., 366(11):5963-5989.

[5] Biermé, H., Estrade, A., and Kaj, I. (2010). Self-similar random fields and rescaled random balls models. J. Theoret. Probab., 23(4):1110-1141.

[6] Bingham, N. H., Goldie, C. M., and Teugels, J. L. (1987). Regular variation, volume 27 of Encyclopedia of Mathematics and its Applications. Cambridge University Press, Cambridge.

[7] Cheng, D. and Liu, P. (2018). Extremes of spherical fractional Brownian motion. Extremes, to appear. arXiv preprint arXiv:1806.02965.

[8] Cohen, S. and Lifshits, M. A. (2012). Stationary Gaussian random fields on hyperbolic spaces and on Euclidean spheres. ESAIM Probab. Stat., 16:165-221.

[9] Durieu, O., Samorodnitsky, G., and Wang, Y. (2017). From infinite urn schemes to self-similar stable processes. Submitted, available at https://arxiv.org/abs/1710.08058.

[10] Durieu, O. and Wang, Y. (2016). From infinite urn schemes to decompositions of self-similar Gaussian processes. Electron. J. Probab., 21:Paper No. 43, 23.

[11] Estrade, A. and Istas, J. (2010). Ball throwing on spheres. Bernoulli, 16(4):953-970.

[12] Gangolli, R. (1967). Positive definite kernels on homogeneous spaces and certain stochastic processes related to Lévy's Brownian motion of several parameters. Ann. Inst. H. Poincaré Sect. B (N.S.), 3:121-226.

[13] Gnedin, A., Hansen, B., and Pitman, J. (2007). Notes on the occupancy problem with infinitely many boxes: general asymptotics and power laws. Probab. Surv., 4:146-171.

[14] Hardin, Jr., C. D. (1981). Isometries on subspaces of $L^{p}$. Indiana Univ. Math. J., 30(3):449-465.

[15] Herbin, E. and Merzbach, E. (2006). A set-indexed fractional Brownian motion. J. Theoret. Probab., 19(2):337-364.

[16] Herbin, E. and Merzbach, E. (2007). The multiparameter fractional Brownian motion. In Math everywhere, pages 93-101. Springer, Berlin.

[17] Istas, J. (2005). Spherical and hyperbolic fractional Brownian motion. Electron. Comm. Probab., 10:254-262.

[18] Istas, J. (2006). On fractional stable fields indexed by metric spaces. Electron. Comm. Probab., 11:242-251.

[19] Istas, J. (2012). Manifold indexed fractional fields. ESAIM Probab. Stat., $16: 222-276$. 
[20] Karlin, S. (1967). Central limit theorems for certain infinite urn schemes. J. Math. Mech., 17:373-401.

[21] Lan, X. and Xiao, Y. (2018). Strong local nondeterminism of spherical fractional Brownian motion. Statist. Probab. Lett., 135:44-50.

[22] Lavancier, F. (2007). Invariance principles for non-isotropic long memory random fields. Stat. Inference Stoch. Process., 10(3):255-282.

[23] Lei, P. and Nualart, D. (2009). A decomposition of the bifractional Brownian motion and some applications. Statist. Probab. Lett., 79(5):619-624.

[24] Lévy, P. (1965). Processus stochastiques et mouvement brownien. Suivi d'une note de M. Loève. Deuxième édition revue et augmentée. Gauthier-Villars \& Cie, Paris.

[25] Marinucci, D. and Peccati, G. (2011). Random fields on the sphere, volume 389 of London Mathematical Society Lecture Note Series. Cambridge University Press, Cambridge. Representation, limit theorems and cosmological applications. [26] Molchan, G. M. (1967). Some problems connected with the Brownian motion of Lévy. Teor. Verojatnost. i Primenen., 12:747-755.

[27] Molchanov, I. and Ralchenko, K. (2015). A generalisation of the fractional Brownian field based on non-Euclidean norms. J. Math. Anal. Appl., 430(1):262278.

[28] Ossiander, M. and Pyke, R. (1985). Lévy's Brownian motion as a set-indexed process and a related central limit theorem. Stochastic Process. Appl., 21(1):133145.

[29] Pipiras, V. and Taqqu, M. S. (2017). Stable non-Gaussian self-similar processes with stationary increments. SpringerBriefs in Probability and Mathematical Statistics. Springer, Cham.

[30] Resnick, S. I. (1987). Extreme values, regular variation, and point processes, volume 4 of Applied Probability. A Series of the Applied Probability Trust. Springer-Verlag, New York.

[31] Richard, A. (2015). A fractional Brownian field indexed by $L^{2}$ and a varying Hurst parameter. Stochastic Process. Appl., 125(4):1394-1425.

[32] Richard, A. (2017). Some singular sample path properties of a multiparameter fractional Brownian motion. J. Theoret. Probab., 30(4):1285-1309.

[33] Robertson, G. (1998). Crofton formulae and geodesic distance in hyperbolic spaces. J. Lie Theory, 8(1):163-172.

[34] Robertson, G. and Steger, T. (1998). Negative definite kernels and a dynamical characterization of property (T) for countable groups. Ergodic Theory Dynam. Systems, 18(1):247-253.

[35] Samorodnitsky, G. and Taqqu, M. S. (1994). Stable non-Gaussian random processes. Stochastic Modeling. Chapman \& Hall, New York. Stochastic models with infinite variance.

[36] Schneider, R. and Weil, W. (2008). Stochastic and integral geometry. Probability and its Applications (New York). Springer-Verlag, Berlin.

[37] Takenaka, S. (1991). Integral-geometric construction of self-similar stable processes. Nagoya Math. J., 123:1-12.

[38] Takenaka, S. (2010). Stable random fields and geometry. In Stability in probability, volume 90 of Banach Center Publ., pages 225-241. Polish Acad. Sci. Inst. Math., Warsaw. 
[39] Takenaka, S., Kubo, I., and Urakawa, H. (1981). Brownian motion parametrized with metric space of constant curvature. Nagoya Math. J., 82:131140.

[40] Čencov, N. N. (1957). Le mouvement brownien à plusieurs paramètres de M. Lévy et le bruit blanc généralisé. Teor. Veroyatnost. i Primenen., 2:281-282.

[41] Venet, N. (2016). On the existence of fractional Brownian fields indexed by manifolds with closed geodesics. arXiv preprint arXiv:1612.05984.

[42] Xiao, Y. (2013). Recent developments on fractal properties of Gaussian random fields. In Further developments in fractals and related fields, Trends Math., pages 255-288. Birkhäuser/Springer, New York.

Zuopeng Fu, Department of Mathematical Sciences, University of Cincinnati, 2815 Commons Way, Cincinnati, OH, 45221-0025, USA.

E-mail address: fuzg@mail.uc.edu

Yizao Wang, Department of Mathematical Sciences, University of Cincinnati, 2815 Commons Way, Cincinnati, OH, 45221-0025, USA.

E-mail address: yizao.wang@uc.edu 